\title{
Challenging Cases Managed by Interventional Radiology
}

\author{
Anna Maria lerardi MD ${ }^{1}$ and Gianpaolo Carrafiello MD PhD ${ }^{1,2}$ \\ 'Radiology Department, Fondazione IRCCS Cà Granda Ospedale Maggiore Policlinico, Via Francesco Sforza, 35, 20122, Milan, Italy \\ ${ }^{2}$ Department of Health Sciences, Università degli Studi di Milano, Milan, Italy
}

With recent advancements in imaging modalities and endovascular techniques, interventional radiology (IR) has become an increasingly popular and often less invasive option for treatment of a number of common emergencies.

The emergency interventional radiology services often require the need for quick decision-making given the clinical status of the patient, availability of resources for early intervention, and availability of alternative therapeutic options.

Interventional radiologists are able to easily interpret the CT scans and thus to optimize the management of patients; furthermore, the IR techniques of embolization and stent-grafting are extremely effective at stemming active haemorrhage.

In some cases, IR proposes itself as the first therapeutical choice over surgery; high chance of postsurgical complications, higher overall mortality rate, and unfitness of some patients for more complex procedures are just few of the many scenarios where IR outshines surgery thanks to the minimal invasiveness of its operations.

Nevertheless, IR requires highly trained specialists to perform efficient and safe procedures and, of course, hi-tech devices that are not currently available in every medical facility. It needs to be said, though, that the promising results and rising effectiveness of IR are encouraging hospitals and radiologists to implement this discipline in their medical routine more and more frequently.

\section{Corresponding author:}

Anna Maria lerardi, MD, Radiology Department, Fondazione IRCCS Cà Granda Ospedale Maggiore Policlinico, Via Francesco Sforza, 35, 20122, Milan, Italy.

Email: amierardi@yahoo.it

(C) 2021 CC BY 4.0 - in cooperation with Depts. of Cardiothoracic/ Vascular Surgery, General Surgery and Anesthesia, Örebro University Hospital and Örebro University, Sweden
The relative novelty of this branch means that often interventional treatments are not standardized or are not yet backed up by an extensive scientific literature; therefore, the interventional radiologist needs to think outside of the box and find solutions that often require a multi-thematical approach (mastery in the medical imaging techniques, different imaging-guided approaches, extensive knowledge of the functioning and usage of devices at their disposal, etc.).

The following series we are proud to present is a collection of challenging cases that were successfully resolved by interventional radiologists. They required multi-disciplinarian choices, often involving different clinicians, but were mostly made possible thanks to the wide knowledge of our specialty, which allowed a fitting solution to be found in the shortest possible time.

\section{Ethics Statement}

(1) All the authors mentioned in the manuscript have agreed to authorship, read and approved the manuscript, and given consent for submission and subsequent publication of the manuscript.

(2) The authors declare that they have read and abided by the JEVTM statement of ethical standards including rules of informed consent and ethical committee approval as stated in the article.

\section{Conflicts of Interest}

The authors declare that they have no conflicts of interest.

\section{Funding}

The authors received no financial support for the research, authorship, and/or publication of this article. 